\title{
Drug Hepatotoxicity in HIV Patients on Highly Active Antiretroviral Therapy [HAART] in Southwest Nigeria
}

\author{
Ayelagbe O. G. ${ }^{1}$, Akerele O. P. ${ }^{2}$, Onuegbu A. J. ${ }^{3}$ Oparinde D. P. ${ }^{1}$ \\ ${ }^{I}$ Departments of Chemical Pathology and ${ }^{2}$ Biomedical Sciences, College of Health Sciences, Osogbo, Ladoke \\ Akintola University of Technology, Ogbomosho, Nigeria. ${ }^{3}$ Department of Chemical Pathology, Nnamdi Azikiwe \\ University, Awka, Anambra State, Nigeria.
}

\begin{abstract}
Hepatotoxicity in HIV is associated with anti-retroviral regimens and hepatitis $B$ and $C$ virus coinfection. Altered liver enzymes activities in HIV mono infected patients yet to commence and those on HAART for at least six months were studied.

45 HIV seropositive patients on HAART for at least six months attending LAUTECH Teaching Hospital, Osogbo, Nigeria, 40 newly diagnosed HIV seropositive patients yet to commence HAART (naive) and $40 \mathrm{HIV}$ negative subjects (control) were recruited. $5 \mathrm{ml}$ venous blood was drawn from each subject. Serum liver function tests were estimated by spectrophotometry. Hepatitis $B$ surface antigen and antibody to hepatitis $C$ virus were determined using ELISA. Data were analyzed statistically and $p<0.05$ was regarded as significant. Hepatotoxicity was defined using a standardized toxicity grade scale.

Total bilirubin, alanine and aspartate aminotransferases (ALT, AST) in HIV-HAART and HIV-naive exceeded the upper limit of normal with lower values recorded in HIV-nä̈ve. There were > 2-fold increase in alkaline phosphatase and AST in HIV-HAART than in controls . Conjugated bilirubin was slightly lower in control subjects than in HIV-HAART and naïve groups. All subjects were negative for HBsAg and anti-HCV.

Even in the absence of HAART, mild hepatotoxicity is seen in HIV patients.
\end{abstract}

Keywords: Antiretroviral Therapy, Hepatotoxicity, HIV, Mono Infection

\section{Introduction}

Abnormal levels of liver enzymes are common among persons infected with human immunodeficiency virus (HIV) and may be caused by multiple factors, including medication toxicity and co infection with hepatitis $\mathrm{B}$ or $\mathrm{C}$ virus [1].

Diagnosis and management may be difficult because of the intricacies of the pathogenic mechanism involved. These include suspected hepatotoxicity related to highly active antiretroviral therapy regimen, immune allergic mechanisms [2], or by the HIV itself influencing a direct damage on the hepatic cells leading to apoptosis and mitochondrial dysfunction of hepatic cells [3].

The period of onset of hepatotoxicity varies between cohorts [4] also patients with HIV often ingest a cocktail of drugs in association with the HAART regimen with the aim of controlling the infection. Some of these alternative and complementary medications have the potential of damaging the liver [5] and therefore pose a high risk for developing drug induced hepatotoxicity [6]. Liver toxicity associated with drugs is part of the spectrum of liver disease [7]. ALT and AST are hepatic enzymes that could be used as markers of hepatocellular injury [8].This study investigates the effect of administration of HAART on the activities of liver enzymes as biochemical markers of hepatotoxicity and possible deleterious effects of these drugs on liver function in HIV/AIDS mono infected patients who have been on HAART for a minimum of six months.

1.1 Materials and methods

1.1.1 Subjects

Forty HIV seropositive subjects (test group) were randomly selected from patients attending the Institute of Human Virology of Nigeria Clinic at LAUTECH Teaching Hospital, Osogbo, Nigeria. The HIV seropositive patients have been on any of the HAART regimens below corresponding with the Nigerian National guidelines for HAART [9] for a minimum of six months :

....Didanosine+Emtricitabine or Lamivudine+Nevirapine or Efavirenz

....Zidovudine+Lamivudine+Nevirapine or Efavirenz

....Stavudine+Lamivudine+ Nevirapine or Efavirenz

Forty newly diagnosed HIV seropositive individuals undergoing therapy at the Institute of Human Virology of Nigeria Clinic of LAUTECH Teaching Hospital but were not yet qualified to commence HAART, according to the Nigerian National guidelines for initiating HAART [9] were designated as "HAART naive" and 
enrolled as the positive control study group. These were followed up every 3 months for possible severity in infection and probability of starting HAART regimen.

Forty subjects attending the General Outpatient Clinic at LAUTECH, Osogbo for routine clinical test and were HIV-negative served as the negative control study groups. All the participants gave their informed consents before the commencement of the study.

\subsubsection{Analytical techniques}

Five $\mathrm{ml}$ of venous blood was collected into plain bottles from each subject. The blood samples were spun in a centrifuge at $4,000 \mathrm{rpm}$ for $5 \mathrm{mins}$. The supernatant was separated into separate screw-cap plain bottles and stored frozen at $2-8^{0} \mathrm{C}$. A semi-structured questionnaire was administered on each participant giving details of history of alcohol consumption, drug intake and general information.

Hepatitis B surface antigen (HBsAg) and antibody to C virus screening were carried out using the rapid antibody technique and ELISA kits. HIV Screening Parallel Testing was done by the Rapid Antibody Test method.

The HIV status of seronegative subjects was confirmed using two HIV rapid antibody test kits Determine ${ }^{\circledR}$ and Unigold ${ }^{\circledR}$ kits. Serum alanine and aspartate aminotransferases, alkaline phosphatase, total and conjugated bilirubin, gamma glutamyl transpeptidase as well as total protein were determined by spectrophotometry.

Osogbo, Nigeria.

Ethical approval was sought from the LAUTECH Teaching Hospital Ethical Approval Committee,

\subsubsection{Statistical analysis}

Data were analysed using statistical package within the Microsoft Excel and SPSS software, USA. Student t-test was used to compare the concentration of serum transaminases and other hepatic parameters of all subjects . $\mathrm{p}$ value less than 0.05 ( $\mathrm{p}<0.05$ ) was considered to be significant.

\section{Results}

The mean ages were $43.3 \pm 6.4,39.9 \pm 7.4$ and $26.9 \pm 4.2$ years for the HIV-HAART, naïve and control subjects respectively. In all, $42.5 \%$ of the subjects were male with the females having the higher percentage. About $47 \%$ of HIV seropositive patients confirmed taking herbal concortions even when they are yet to commence HAART regimen.

Table 1: Serum biochemical parameters in HIV negative subjects and HIV positive subjects on HAART $($ Mean \pm SD)

\begin{tabular}{|l|l|l|c|}
\hline Parameter & HIV negative subjects & HIV positive subjects on HAART & p-value \\
\hline ALT(U/l) & $6.7 \pm 2.0$ & $24.9 \pm 5.4$ & $* 0.00$ \\
\hline AST(U/l) & $9.9 \pm 2.3$ & $27.4 \pm 6.3$ & $* 0.00$ \\
\hline ALP(U/l) & $22.1 \pm 6.9$ & $49.1 \pm 17.9$ & $* 0.00$ \\
\hline GGT(U/l) & $9.9 \pm 3.9$ & $12.0 \pm 4.5$ & 0.09 \\
\hline Tot bil(mg/dL) & $10.0 \pm 2.8$ & $18.9 \pm 3.4$ & $* 0.00$ \\
\hline Conj bil(mg/dL) & $2.4 \pm 0.9$ & $4.5 \pm 1.2$ & $* 0.00$ \\
\hline Tot Protein(g/dL) & $5.7 \pm 2.1$ & $5.4 \pm 3.0$ & 0.12 \\
\hline
\end{tabular}

*significant at $\mathrm{p}<0.05$, Tot bil - total bilirubin, conj bil. - conjugated bilirubin, GGT-gamma glutamyl transpeptidase

The activities of serum transaminases and bilirubin (total and conjugated) were compared in Table 1. This showed significant increases greater than 1.25 -2.5 x upper limit of normal (ULN) in ALT, AST (Grade 1 hepatotoxicity), ALP, total and conjugated bilirubin levels in HIV patients on HAART when compared with the HIV seronegative subjects that exhibit Grade 0 hepatotoxity (that is less than $1.25 \mathrm{ULN}$ ). Normal ranges relative to upper limit of normal for ALT, AST and total bilirubin $=12 \mathrm{U} / \mathrm{L}[10,11,12]$.

Table 2: Serum liver enzymes and bilirubin values in HAART naive subjects and HIV positive subjects on HAART $($ Mean \pm SD)

\begin{tabular}{|l|l|l|c|}
\hline Parameter & HAART naive subjects & $\begin{array}{l}\text { HIV positive subjects on } \\
\text { HAART }\end{array}$ & p-values \\
\hline ALT(U/l) & $11.8 \pm 4.9$ & $17.9 \pm 5.4$ & $* 0.01$ \\
\hline AST(U/l) & $16.2 \pm 6.0$ & $27.4 \pm 6.3$ & $* 0.00$ \\
\hline ALP(U/l) & $14.9 \pm 5.1$ & $49.1 \pm 17.9$ & $* 0.00$ \\
\hline GGT(U/l) & $11.1 \pm 3.3$ & $12.0 \pm 3.9$ & 0.11 \\
\hline Tot bil(mg/dL) & $13.9 \pm 3.2$ & $18.9 \pm 3.4$ & $* 0.00$ \\
\hline Conj bil(mg/dL) & $3.1 \pm 1.4$ & $4.5 \pm 1.2$ & $* 0.01$ \\
\hline Tot Protein(g/dL) & $5.2 \pm 2.2$ & $5.4 \pm 3.0$ & 0.11 \\
\hline
\end{tabular}


* significant at $\mathrm{p}<0.05$

Liver enzymes, total and conjugated bilirubin were significantly increased in HIV positive patients on HAART compared with the HAART naïve subjects. There was $>3$-fold increase in the value obtained for serum ALP and a near two fold increase in AST in patients on HAART. There were no significant differences in gamma glutamyl transpeptidase and total protein in both groups. (Table 2).

Table 3: Serum liver enzymes and bilirubin values in HAART naive subjects and HIV negative subjects (Mean \pm SD)

\begin{tabular}{|l|l|l|c|}
\hline Parameter & HAART naive subjects & HIV negative subjects & p-values \\
\hline ALT(U/l) & $11.8 \pm 4.9$ & $6.7 \pm 2.0$ & $* 0.00$ \\
\hline AST(U/l) & $16.2 \pm 6.0$ & $9.9 \pm 2.3$ & $* 0.00$ \\
\hline ALP(U/l) & $14.9 \pm 5.1$ & $22.1 \pm 6.9$ & $* 0.01$ \\
\hline GGT(U/l) & $11.1 \pm 3.3$ & $9.9 \pm 3.9$ & 0.10 \\
\hline Tot bil(mg/dL) & $13.9 \pm 3.2$ & $10.0 \pm 2.8$ & $* 0.00$ \\
\hline Conj bil(mg/dL) & $3.1 \pm 1.4$ & $2.4 \pm 0.9$ & 0.06 \\
\hline Tot Protein(g/dL) & $5.2 \pm 2.2$ & $5.7 \pm 2.1$ & 0.08 \\
\hline
\end{tabular}

*The mean difference is significant at $\mathrm{p}<0.05$

Table 3 showed significant differences in the values of liver enzymes and total bilirubin but not in conjugated bilirubin levels in HIV-HAART patients and HIV seronegative subjects.

\section{Discussion}

Data from this study showed that the activities of the transaminases in patients on HAART exceeded the upper limit of normal compared with the HIV patients that were not on HAART and the HIV negative subjects. These findings concur with previous studies $[13,14]$ that reported a characteristic increase in liver transaminases as a result of administration of HAART on patients. An elevation in the activity of alkaline phosphatase and a concomitant surge in the total bilirubin level as observed in the HAART patients in this study is suggestive of a near cholestatic condition and it could be hypothesized that jaundice may develop with time if adequate attention is not paid to the rising bilirubin values [12].

Patients on HAART were observed to exhibit degree 1 hepatotoxicity [10]. Degree 1 hepatotoxicity is a weak form of hepatotoxicity which do not present as an aggravating condition [15] and so does not require the withdrawal of the ARVs in question, whereas the negative control group showed degree 0 hepatotoxicity which is normal for any individual [15].

The observed elevation in the serum transaminases in sero-positive patients in this study may be caused by the HIV infection itself or be as a result of the presence of opportunistic infections which stimulates an immunological response by hepatic phagocytes against the infection [3]. This is further supported by another study which reported that apart from HAART-derived hepatotoxicity, some liver diseases are often associated with HIV infection leading to increased transaminases [16]. However, immunological tests were not performed on the patients

studied. Nevertheless, even in the absence of HAART, HIV/AIDS patients have the tendency of having elevated liver enzymes, although this does not necessarily imply a drug-induced hepatotoxicity dependent on HAART.

In the course of interaction with the HIV patients, some of them confirmed the use of local, herbal concoctions and other sources of alternative therapies as additional means of combating the viral load and restoring immune functions. This is compounded by their cultural and religious beliefs. Some of these locally manufactured concoctions are potentially toxic to the liver, and their intake may further hamper the ability of the liver to detoxify toxins in its sinusoids [17]. Hence the detoxifying ability of the liver and its ability to conjugate drugs are gradually lost. For instance garlic - an active ingredient in the herbal concoctions -may stop saquinavir - a protease inhibitor from working properly [18]. It is worthy of note that non-adherence to drugs regimen and treatment of opportunistic infections with over the counter drugs have been indicted to further compound the problem of increased transaminase level in HIV patients. This is backed up by a study on drug interactions in HIV patients that reported a significant association between the administration of sulfonamides, antituberculosis agents and grade 1 hepatotoxicity in children [19]. Common reasons identified for patient's nonadherence in this community include the

cost of therapy, medication side effects, non-availability of antiretroviral (ARV) drugs and the stigmatization of people living with HIV/AIDS [18].

Severe ARV induced hepatotoxicity was not encountered during the course of this study probably because administration of the regimens were well tolerated by the patients. 


\section{Conclusion}

Hepatotoxicity is a growing problem among HIV patients on HAART and could be manifested within six weeks of drug intake. Therefore it is important to monitor liver function at periodic intervals in the course of drug administration. Administration of a fixed dose combination made up of a single dose or capsule will reduce preference for alternative herbal concoction which poses a risk to normal hepatic function. A further study would be undertaken to establish the effect of a long-term administration of HAART on liver functions and a liver biopsy to investigate changes in the histological architecture of a hepatotoxic compared to a normal functioning liver.

\section{References}

[1]. M Egger, M May, G Chene, B Hirscel, W Huber, and T S Li, Prognosis of HIV-1 infected patients starting highly active antiretroviral therapy: A collaborative analysis of prospective studies, Lancet; 60, 2002,119-129.

[2]. P CaCoub , P Stanislas, C Perronne, and ANRS HCO2 - Ribavic study Team, Management of the Co-infected Patient with Human Immunodeficiency Syndrome on Course of Hepatitis C Virus Infection: A Meta-Analysis, Infectious Disease in Clinical Practice, 15(1),: 2004, 38-48.

[3]. J A Mata-Marín, J Gaytán-Martínez, H B Grados-Chavarría , J Fuentes-Allen , C I Arroyo-Anduiza and A Alfaro-Mejía, Correlation between HIV viral load and aminotransferases as liver damage markers in HIV infected naive patients: a concordance cross-sectional study. Virology Journal, 6, 2009,181.

[4]. I Sanne, H Mommeja-Marin, J Hinkle, J A Bartlett, M M Lederman , and G Maartens, Severe hepatotoxicity associated with nevirapine use in HIV-infected subject. Journal of Infectious Disease, 191, 2005,825-9.

[5]. V J Navarro, and J R Senior, Drug related hepatotoxicity, National England Journal of Medicine, 354, 2006,731-9.

[6]. J D Lewis, and B L Strom, Balancing safety of dietary supplements with the free market. Annals of Internal Medicine, 136, 2002,616-8.

[7]. M Bonacini, and M Pouti, Hepatitis $\mathrm{C}$ in patients with human immunodeficiency virus infection. Diagnosis, natural history, metaanalysis of sexual transmission, and therapeutic issues. Archives of Internal Medicine, 160, 2000, 3365-3373.

[8]. B Zechini, Z Pasquazzi, and A Aceti, Correlation of serum aminotransferases with hepatitis C RNA levels and histological findings in patients with chronic hepatitis $\mathrm{C}$ : the role of serum aspartate aminotransferase in the evaluation of disease progression. European Journal of Gastroenterology Hepatology, 16, 2004,891-896.

[9]. Guidelines for the use of antiretroviral drugs in Nigeria, The Federal Ministry of Health in conjunction with WHO, NACA, UNAIDS, and DFID; The Honourable Minister, Federal Ministry of Health, Abuja, Nigeria, 2005.

[10]. F W Wit, G J Weverling, J Weel, S Jurriaans and J M Lange, Incidence of and risk factors for severe hepatotoxicity associated with antiretroviral combination therapy, Journal of Infectious Disease, 186, 2002, 23-31.

[11]. N Kontorinis, and D Dieterich, Hepatotoxicity of antiretroviral therapy. AIDS Review, 5(1), 2003, 36-43.

[12]. M S Sulkowski, D L Thomas, R E Chaisson, and R D Moore, Hepatotoxicity associated with antiretroviral therapy in adults infected with human immunodeficiency virus and the role of hepatitis $\mathrm{C}$ or B virus infection, Journal of American Medical Association, 283(1),2000,74-80.

[13]. U Spengler, M Lichterfeld, and J K Rockstroh, Antiretroviral drug toxicity -a challenge for the hepatologist? Journal of Hepatology, 36(2), 2002,283-94.

[14]. J A Raúl, R Mercedes, A Fernández-Castañer, S López Ortega , M C López-Vega, and M I Lucena, (2007). Assessment of druginduced hepatotoxicity in clinical practice: A challenge for gastroenterologists. World Journal of Gastroenterology, 13(3), 2007, 329-340.

[15]. S Pol, P Lebray, and A Vallet-Pichard, HIV infection and hepatic enzyme abnormalities: Intricacies of the pathogenic mechanisms, Clinical Infectious Diseases; 38 Suppl 2, 2004, S65-72.

[16]. P D Mayne, Clinical chemistry in diagnosis and treatment (6th ed. UK: ELBS Chapter 14, 1994)

[17]. S Piscatelli, The effect of garlic supplementation on the pharmacokinetics of saquinavir. Clinical Infectious Disease, 34(2), 2002, 234-238.

[18]. I Shah, Adverse effects of anti retroviral therapy in HIV-1 infected children. Journal of Tropical Pediatrics. 52(4), 2005,244-248.

[19]. E Monjok, A Smesny ,I Okokori, O Mgbere, and Essien E, Adherence to anti retroviral therapy in Nigeria: an overview of research studies and implication for policy and practice in HIV/AIDS. Research and palliative care, 2, 2010, 69-76. 